\title{
Faint Photometry with the HST Wide Field Camera
}

\author{
S.M.G. Hughes ${ }^{1}$ \\ ${ }^{1}$ Palomar Observatory 105-24, California Institute of Technology, \\ Pasadena CA 91125, USA
}

\begin{abstract}
A series of 40 HST WFC exposures in $v$ (F555W) and $i$ (F785LP) have been obtained of two fields in M81, in order to search for Cepheid variables. This has provided an ideal opportunity to compare how the photometry programs DoPHOT and DAOPHOT cope with undersampled images, and present our 'best solution' to the problem of reducing WFC photometry for faint stars in crowded fields.
\end{abstract}

Although there are major obvious advantages in using WFC images (such as 0.1 arcsec resolution), measuring stellar magnitudes with WFC poses several problems in addition to those normally associated with ground-based CCDs. Not only is there a much higher cosmic ray count and the image cores are undersampled, but the aberrated optics of HST give rise to a 3 arcsec halo and a variable point spread function (PSF), and the flat fields (obtained from earth-streaked images) are not ideal. In addition, since early 1992 the chips have been seen to suffer from periodic contamination which creates a measles effect, but happily for $v$ and $i$-type projects, is most pronounced at short wavelengths.

Fortunately, most of these problems should be fixed with the installation of WFPC 2 at the end of 1993, which is designed to not only correct the optical aberrations, but will have better detectors and an internal calibration ("flat-field") lamp.

However, even with the current problems, it is still possible to do faint photometry, and one of the major projects of HST, to calibrate several extragalactic distance indicators to better than $10 \%$ using Cepheids to measure distances to galaxies out to the Virgo group, is about to complete its first phase, by finding a large sample of Cepheids in M81 (Mould 1992).

Photometry programs that fit conventional Gaussian-like PSFs are inappropriate to WFC images, due to the undersampling of the diffraction-limited core spikes. Two popular photometry packages that have had their psf routines suitably modified are DAOPHOT II (Stetson 1991, which fits a Lorentz function) and DoPHOT 2.0 (based on Mateo \& Schecter's 1989 DoPHOT program) with its pseudo-Gaussian routine replaced by a routine written by Abi Saha (STScI), which is designed to match the PSF shape of the WFC cores. The performance of these two packages have been compared by adding $5 \times 100$ randomly positioned pseudo-stars, covering a range of known magnitudes with photon (Poisson) noise included (using ADDSTAR in DAOPHOT II) to five identical medianed images of an M81 field (derived from $8 \times 900 \mathrm{sec}$ F555W exposures). The PSF function used by ADDSTAR to create the pseudo-stars was derived from a $7 \times 7$ grid of PSF stars generated by the TINYTIM code (Krist 1992, STScI, private communication). The differences between the measured and actual magnitudes are plotted in Figure 1, along with the standard deviation in each of several magnitude bins. Clearly, DAOPHOT does much 
better at most magnitudes, although this may be partly due to the use in DAOPHOT of the same PSF function that was used to create the pseudo-stars, and also because the standard version of DoPHOT 2.0 does not allow for a quadratically-varying PSF. However, at the magnitude of Cepheids ( $\sim 24$ at the distance of M81), each is of comparable performance, giving errors of $\sim 0.2 \mathrm{mag}$.

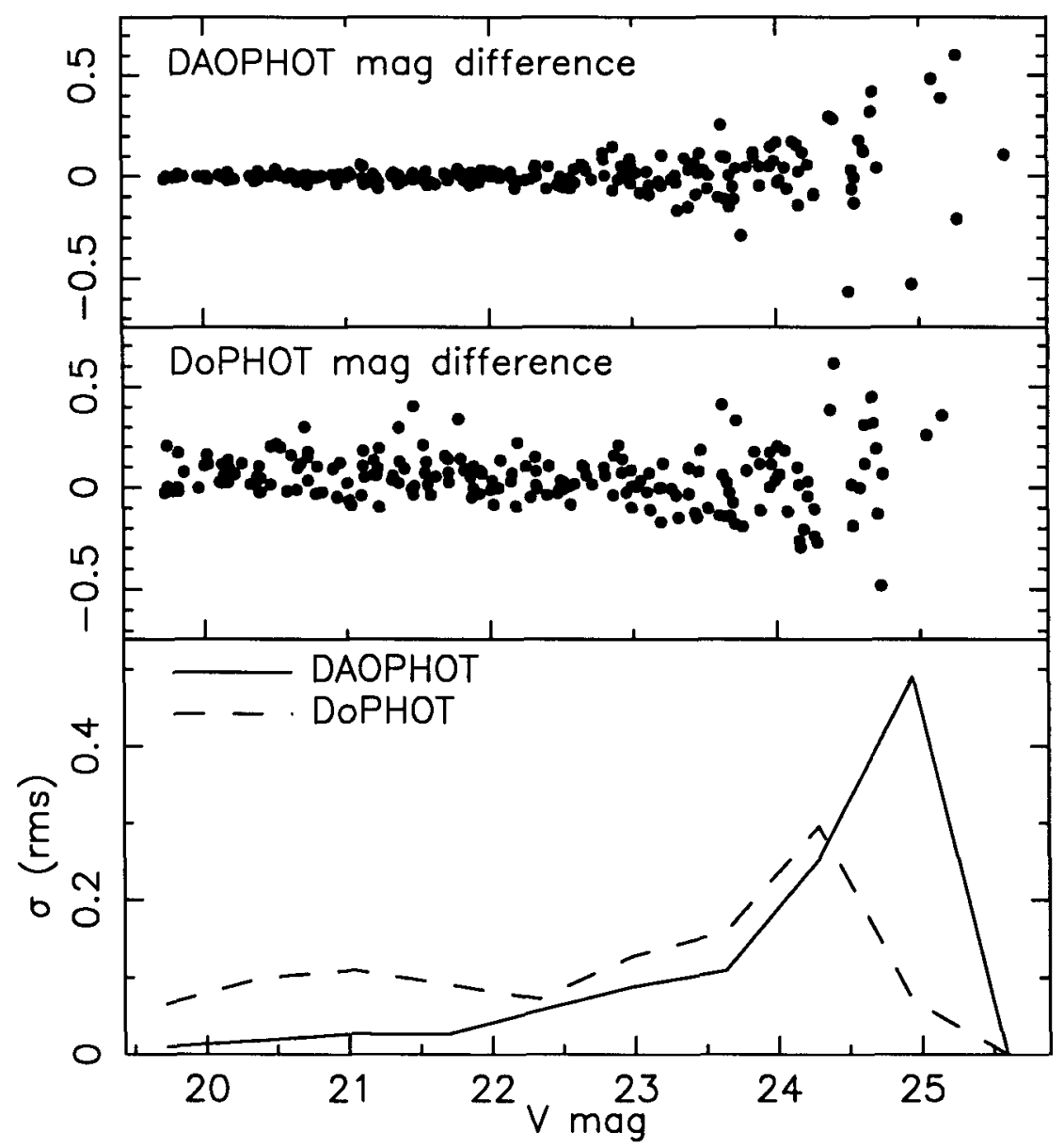

Figure 1 The differences between the measured and known magnitudes of pseudo-stars, obtained by DAOPHOT II (top panel), and DoPHOT 2.0 (middle panel). The bottom panel shows the standard deviation of these differences per magnitude bin, as a function of approximate $V$ magnitude.

However, even better results are now being obtained by Stetson (1992, private communication) using ALLFRAME, a code based on DAOPHOT II, but which simultaneously analyses all common frames. Using ALLFRAME, 30 Cepheid candidates have been found in the two M81 fields. A further 8 frames will be obtained in Cycle 2 to confirm 
and refine their periods.

Having obtained precise relative photometry, the data need to be calibrated. Initial calibration of the data has been made from Palomar 200-inch COSMIC frames obtained by Jeremy Mould. Here the main problem has been that about half the objects that appear stellar in the COSMIC frames are in fact extended (probably HII regions) in the WFC frames, and so all extended objects have to be eliminated before matching with the ground-based photometry. Current calibration of the zero-point has an uncertainty of 0.2 mag, but it is possible this will improve with better flat-fielded WFC data and modelling the vignetting across the WFC field (Holtzman et al 1991).

\section{Acknowledgements}

I am greatly indebted to Peter Stetson for valuable advice and for guiding me through the intricacies of DAOPHOT, to Abi Saha for sending me both the UNIX version of DoPHOT 2.0 and his WFC PSF routine, to the members of the $\mathrm{H}_{0}$ team, in particular Jeremy Mould, Myung Lee, Barry Madore and Wendy Freedman, and to Mario Mateo for helpful advice with DoPHOT.

\section{References:}

Holtzman J.A. et al, 1991 AJ 369, L35.

Mateo M., Schecter P. 1989, in Proc of 1st ESO ST-ECF Data Analysis Workshop, eds. P.J.Grosbol: F.Murtagh and R.H.Warmels (Garching:ESO), p69.

Mould J.R. 1992, Science with the HST, ECF conference July 1992, Sardinia, in press.

Stetson P.B. 1991 in 3rd ESO/ST-ECF Data Analysis Workshop, Garching April 1992, ESO

Conference and Workshop proceedings No.38, eds. P.J.Grosbol and R.H.Warmels, p187.

\section{Discussion}

M. Taylor: I realise that using an internal calibration lamp will probably improve the flatfielding problems significantly, but won't you still need to do external flats to determine the characteristics outside the camera optics?

T.J. Kreidl: Have you tried deconvolution, well aware that there may be fux-conservation problems, to see if this might help the situation?

Hughes: Nick Weir (Caltech) has been experimenting with a new version of his Maximum Entropy code, that should be capable of conserving flux. In the meantime, we intend using deconvolved images to identify faint and crowded images, whose positions will then be used in ALLFRAME to derive photometry from the original images.

E. Budding: Could you explain the term "internal flat-fielding"? Also, how soon is it going to be before this whole flat-fielding business can be ignored at the $0.1 \%$ level?

Hughes: WFPC2 will have an internal calibration module that will be used to monitor the stability of the CCDs' response, but to obtain a true calibration (to remove the effects 
of telescope plus WFC optics plus detector response) external flat-fields will still be needed. As to when we will no longer need flat-fields, that depends not only on uniform CCD response, but also on telescope optics, which currently are certainly not uniform over large fields of view, nor stable over time, due to thermal and gravitational flexure and deteriorating optical surfaces.

R.R. Shobbrook: In spite of the problems, you still have a distance to M81 far more accurate than before this work?

Hughes: Yes, that is certainly true. The aim is to measure galaxy distances to better than $5 \%$. Since we have $\sim 16$ good Cepheid candidates from 24 epochs (includes cycle 2 time), these results show that we should be able to measure a reliable distance to an internal accuracy (ignoring reddening and $\mathrm{P}, \mathrm{L}$ zero-point uncertainties) of $<2 \%$ for M81, but eventually we intend to observe galaxies out to Virgo. 